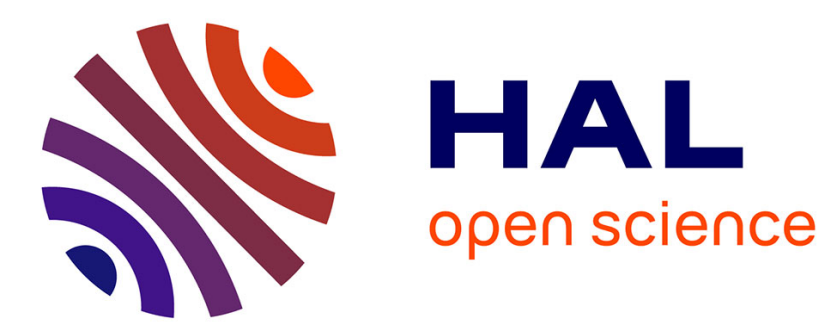

\title{
Strategic Product Design under Duopoly
}

Didier Laussel

\section{To cite this version:}

Didier Laussel. Strategic Product Design under Duopoly. Annals of Economics and Statistics, 2018, 131, pp.25. 10.15609/annaeconstat2009.131.0025 . hal-01992047

\section{HAL Id: hal-01992047 https://hal-amu.archives-ouvertes.fr/hal-01992047}

Submitted on 24 Jan 2019

HAL is a multi-disciplinary open access archive for the deposit and dissemination of scientific research documents, whether they are published or not. The documents may come from teaching and research institutions in France or abroad, or from public or private research centers
L'archive ouverte pluridisciplinaire HAL, est destinée au dépôt et à la diffusion de documents scientifiques de niveau recherche, publiés ou non, émanant des établissements d'enseignement et de recherche français ou étrangers, des laboratoires publics ou privés.

\section{(c)(1)}

Distributed under a Creative Commons Attribution| 4.0 International License 


\title{
STRATEGIC PRODUCT DESIGN UNDER DUOPOLY
}

\author{
DIDIER LAUSSEL ${ }^{a}$
}

Two duopolists first decide in which proportions to incorporate in their product two different Lancasterian characteristics and then compete in quantities or prices. In the Cournot case, minimum differentiation obtains at equilibrium whatever the de-gree of substituability between the characteristics. In the Bertrand one, the equi-librium depends crucially on the degree of substituability/complementarity between the two characteristics. Maximal differential obtains if and only if the characteristics are strong enough substitutes. On the contrary as characteristics become closer and closer complements one obtains in the limit a minimal differentiation result.

Keywords: Horizontal Product Differentiation, Lancasterian Characteristics.

\section{INTRODUCTION}

In his "new approach to consumer theory", Lancaster (Lancaster, 1966, 1971; Lancaster Kelvin, 1975) argued that each good is a combination of intrinsic properties which he labeled "characteristics". He considered that this is what makes butter and margarine good substitutes or automobiles and gasoline intrisically complementary. He gave the example of a meal which may possess nutritional and aesthetic characteristics "in different relative proportions".

According to this approach, product design amounts to determine, when assembling the product, the relative proportions of the characteristics which enter it. It has been analyzed by Johnson and Myatt (2006) ${ }^{1}$ from the point of view of a monopolist who has to choose the weights of $n$ characteristics in its product in order either to maximize or on the contrary to minimize dispersion of consumers valuations ${ }^{2}$. As far as we know strategic product design, the choice of the relative proportions of the different characteristics in their respective products by oligopolists prior to competing in prices or quantities, has never been explicitly considered as such in the literature which has followed the seminal paper of Hotelling (1990) and its reformulation fifty years later by d'Aspremont, Gabszewicz, and Thisse (1979).

These two models could indeed be alternatively viewed as a spatial model dealing with location of firms in a physical space or as an horizontal differentiation model in which the firms choose locations in an abstract product space. In the first case the firms supply the same products but may be located at different places while, in the second, they are located at the same place but may supply different products. This equivalence was formally proved by Peitz (1997) who proposed "a class of models which can be written as models à la Lancaster as well as models à la Hotelling" and in which the two characteristics are always perfect substitutes (and consumers have unit demands). These models share some common features: the demand is perfectly inelastic and the firms compete

\footnotetext{
${ }^{a}$ Aix Marseille Université (Aix Marseille School of Economics), CNRS \& EHESS, didier.lausseleuniv-amu.fr

${ }^{1}$ We thank an anonymous referee for having drawn our attention to this paper.

'The authors' basic finding is that profits are a convex function of the dispersion of consumers' valuations.
} 
in prices (Bertrand equilibrium). The main conclusion which emerges from d'Aspremont, Gabszewicz, and Thisse (1979), is the following: in a two-stage game, where firms choose locations first and then compete in prices, firms never agglomerate. They disperse maximally in order to soften the second-stage price competition ${ }^{3}$.

The models which have challenged the robustness of the dispersion result are spatial models and for most if not all of them cannot be naturally reinterpreted as dealing with choices of horizontal product differentiation. In De Fraja and Norman (1993), who remove the assumption of demand inelasticity, the degree of product differentiation is exogenously fixed. Anderson and Neven (1991), Hamilton, Thisse, Weskamp, et al. (1987) and Gupta, Pal, and Sarkar (1997), who deal with location choice under Cournot competition, consider homogenous products in a pure spatial model. Hamilton, Thisse, Weskamp, et al. (1987) deal with spatial discrimination as well as De Palma, Labbé, Thisse, and Norman (1986) who compare mill and uniform delivered spatial pricing. De Palma, Ginsburgh, Papageorgiou, and Thisse (1985) consider that consumers at each point of the Hotelling line have heterogenous unobservable preferences, an assumption which leads to a minimum differentiation result but is meaningless outside a spatial framework.. This spatial feature is all the more present in the several recent papers (Alcacer and Chung (2007), Colombo and Dawid (2014) among others) which have investigated the role of knowledge spillovers in the decision of firms to locate in isolation or in a cluster.

Our purpose is on the contrary to analyze horizontal product differentiation or, more precisely, strategic product design: we adopt a Lancasterian approach to product differentiation which allows to obtain new insights in this field. We suppose that consumers derive utility from the consumption of two characteristics of the products they buy, these characteristics being more or less complementary or substitutable from the consumers' point of view ${ }^{4}$, and that two duopolists independently determine in which relative proportions these characteristics will be present in their respect products. Doing that, they anticipate the consequences of these choices on the subsequent competition in quantities or prices. Though Lancaster himself did not comment on the substitutability or complementarity between characteristics, this feature turns out to be important for strategic product design and also empirically relevant. Houses may be a good example of complementary characteristics. In order to focus on horizontal differentiation, let's consider houses with the same building cost, i.e. roughly with the same surface, and two characteristics: the number of bedrooms and the number of bath rooms. These two characteristics are clearly complementary (if not perfectly so) since very few people would like to have for instance more bathrooms than bedrooms. This is all the more true if one replaces bathrooms by kitchens. A good example of substitutable characteristics may be newspapers. Let us consider for instance as the two characteristics ${ }^{5}$ culture (the literary and film current events and so on) and entertainment (the current events of the celebrity). The former is certainly for most readers a substitute for the latter. Some studies have investigated the substitutability / complementarity between the informational characteristics of agricultural products, without

\footnotetext{
${ }^{3}$ This conclusion has been questioned by Irmen and Thisse (1998) who showed that in a $n$-dimensional space with $n>2$ "Hotelling was almost right", i.e. the principle of minimum differentiation holds in all but one dimension.

${ }^{4}$ Among other things, this differentiates this paper from Peitz (1997) who assumed that characteristics are perfect substitutes.

${ }^{5}$ That could be as well sport and politics.
} 
however drawing consequences of the results for strategic product design by competing firms. Gracia, Barreiro-Hurlé, and Galán (2014) on the Spanish eggs market showed that origin (local) and organic claims are complementarity for the larger market segment ("origin preference") and substitutable for the smaller one ("production method preference"). In the same spirit, Meas, Hu, Batte, Woods, and Ernst (2014) showed in a US study on blackberry jam that organic and local production claims were substitutes while the "small farm" attribute appeared also to be a substitute for the organic and local attributes. The consequences for strategic product design have not yet been drawn.

In our model, each firm has then to choose in a first stage of the game what exact mix of the characteristics will be embodied in each unit of their output. Consumers' utility then depends continuously on the quantities of the two goods which they buy and demands vary themselves continuously as prices vary. They subsequently compete in quantities or in prices in the second stage of the game. This yields a simple and tractable model of product differentiation which is as close as possible to the Hotelling's spatial differentiation one (horizontal differentiation choices are represented by a point on the unit segment) while incorporating important additional features: non mutually exclusive consumptions of the goods, a non inelastic aggregate demand and the possibility for the competing firms to choose to supply complementary products. In particular, having a non fixed aggregate market size allows to study the case of Cournot competition.

\section{Main results and related literature.}

In the Cournot case, the main result is minimum product differentiation: at the Nash equilibrium of the game, the two firms choose to supply identical products, i.e. products incorporating the same mix of characteristics.

In the Bertrand case, our main finding is that the equilibrium depends crucially on the degree of substitutability/complementary between the two characteristics, which turns out to be the key parameter. Maximal differentiation (pure "niche" strategies) obtains if and only if the characteristics are strong enough substitutes. To refer to our second example above, newspapers will be differentiated at equilibrium, dealing mainly with culture or with entertainment. On the other side, as characteristics become less substitutable or more complementary, the firms differentiate less and less their products, approximating in the limit a minimal differentiation result. To refer to the houses example, new houses for sale will have approximately the same ratio between the number of bedrooms and the number of bathrooms (or kitchens). This differs from results obtained in the Hotelling framework by d'Aspremont, Gabszewicz, and Thisse (1979) but also from the generalization by Economides et al. (1986), since, for a large range of values of the degree of substitutability/ complementarity between parameters, we have neither maximal nor minimal differentiation but increasing differentiation as the characteristics become more and more substitutable. The intuition also is new: the direct, negative, effect on the demand for the firm's product of offering a more differentiated good is increasing in the degree of complementarity between the characteristics. When the characteristics are strongly complementary, a change in the firm's characteristics mix away from a balance between the two reduces the demand drastically ${ }^{6}$. This induces the firms to differentiate less their prod-

\footnotetext{
${ }^{6}$ Considering houses with four rooms, no one would probably buy a housewith no kitchen or with two kitchens.
} 
ucts in equilibrium. When, on the contrary, they are very substitutable, the same change does not modify importantly the demand. As far as we know this key role of the degree of substitutability between characteristics in firms' strategic product differentiation choices had never been stressed before in the literature.

Globally our results are in line with corresponding ones in the existing spatial literature for the Cournot case. For instance, Pal (1998), for the linear city case ${ }^{7}$, and De Fraja and Norman (1993), who assumed differentiated goods and elastic demands, concluded that firms agglomerate at the center Gupta, Pal, and Sarkar (1997) provided conditions for this very result to hold when the distribution of consumers is not uniform.

Our results for the Bertrand case are more original. Close ones are in Economides et al. (1986) who generalized d'Aspremont, Gabszewicz, and Thisse (1979) by considering consumers' transportation costs which are a function $d^{\alpha}$ of the distance from the firm where $\alpha$ may differ from 2 . He showed that, when price equilibria exist, minimal differentiation is never an equilibrium outcome, while maximal differentiation obtains only for $\alpha \geq 5 / 3$. The degree $\delta$ of substitutability/ complementarity between the Lancasterian characteristics plays here a role similar to $\alpha$ in Economides' analysis to generate a range of different differentiation outcomes. Our model, however, allows, contrary to Economides, for the existence of a pure-strategy price equilibrium for all possible values of $\delta$. It is definitely not spatial and does not suppose an inelastic fixed aggregate demand. Moreover, it approximates in the limit the minimum differentiation outcome for the perfectly complementary characteristics case. Irmen, Thisse, et al. (1998) have studied the case of Bertrand competition and a multi-characteristics space, showing that "Hotelling was almost right" since firms choose to differentiate maximally only in the dominant characteristic and minimally in all the other ones ${ }^{8}$. However, their model is an extension of Hotelling's spatial model with (quadratic) transportation costs to a multidimensional space where the "characteristics" which are considered are not Lancasterian ones but simply mean dimensions in Hotelling's space ${ }^{9}$.

Our approach bears also some resemblance to a recent paper by Brander and Spencer (2015) in which the two firms choose in a first stage the degree of differentiation of their products by investing in advertising before competing in a second stage either in quantities or in prices. Brander and Spencer not surprisingly concluded that Bertrand firms always differentiate more their products than do their Cournot counterparts, when the latter do differentiate them. More surprisingly, they showed that Bertrand firms, since they differentiate more, may end up charging higher prices and earning higher profits than Cournot ones. Our approach, which has Lancasterian microeconomic foundations and allows for a much richer set of possible demand parameters, does not confirm the latter result: Bertrand firms' prices and profits are smaller than Cournot's ones for all possible values of $\delta$.

\footnotetext{
${ }^{7}$ While they locate equidistantly on the circular city.

${ }^{8}$ A close paper is Neven and Thisse (1989) who analyze price competition in a model where firms differentiate both horizontally and vertically. At equilibrium they differentiate maximally along one dimension and minimally along the other.

${ }^{9}$ Notice that, in a Lancasterian model with $n$ characteristics, firms' horizontal differentiation choices are choices of points in a $n-1$ dimensions space.
} 


\section{THE MODEL}

Let us consider a representative consumer (a continuum of identical consumers would be as good) with linear-quadratic preferences defined over the quantities $C_{A}$ and $C_{B}$ of two Lancasterian "characteristics" which he/she consumes and the quantity $Q_{0}$ of a "numeraire" good

$$
U=\left(C_{A}+C_{B}\right)-\frac{1}{2}\left(C_{A}^{2}+C_{B}^{2}\right)-\delta C_{A} C_{B}+Q_{0}
$$

where $\delta \in(-1,1)$. The two characteristics may be substitutes, if $\delta>0$, or complements, if $\delta<0$. The limit case of perfect substitutes corresponds to $\delta=1$, where $U$ is then only function of $\left(C_{A}+C_{B}\right)$, namely $U=\left(C_{A}+C_{B}\right)-\frac{1}{2}\left(C_{A}+C_{B}\right)^{2}$. In the limit case where $\delta=-1$, we shall speak of pure complements, since both the own and cross priceelasticities of demand go to minus infinity as $\delta \rightarrow-1$ (see equations (7) below). Notice that as $\delta \rightarrow-1, U \rightarrow\left(C_{A}+C_{B}\right)-\frac{1}{2}\left(C_{A}-C_{B}\right)^{2}+Q_{0}$.

Consuming one unit of good 1 (produced by firm 1) provides $1-a$ units of characteristic $A$ and $a$ units of characteristic $B$. Consuming good 2 (produced by firm 2) provides $b$ units of characteristic $A$ and $1-b$ units of characteristic $B$. Hence the choice of $a$ by firm 1 and of $b$ by firm 2 is the choice of the proportions of the two characteristics in their product ${ }^{10}$. It can be conveniently represented as the choice of a position on the interval $[0,1]$. It follows that

$$
\begin{aligned}
& C_{A}=(1-a) Q_{1}+b Q_{2}, \\
& C_{B}=a Q_{1}+(1-b) Q_{2} .
\end{aligned}
$$

Substituting for $C_{A}$ and $C_{B}$ in (1) their values from (2), we obtain the consumer's utility as a function of the quantities consumed of the three goods:

$$
u\left(Q_{0}, Q_{1}, Q_{2}\right)=Q_{1}+Q_{2}-\frac{1}{2}\left(G Q_{1}^{2}+D Q_{2}^{2}\right)-E Q_{1} Q_{2}+Q_{0}
$$

where $G=1-2 a(1-a)(1-\delta), E=(a+b+\delta(1-a-b)-2 a b(1-\delta)), D=$ $1-2 b(1-b)(1-\delta)$. Notice that $G$ and $D$ are always non-negative ${ }^{11}$.

The utility function (3) can be usefully compared to the similar, linear-quadratic, ones used by Bowley (1924), Dixit (1979), Singh and Vives (1984) or Tremblay and Tremblay (2011) in which the coefficients of the second-order polynomial in $Q_{1}$ and $Q_{2}$ are exogenously given. Here they are here determined endogenously by the mixes of characteristics $a$ and $b$ selected by the two competing firms. Finally, Brander and Spencer (2015) use as well a linear-quadratic utility function, with $G=D=1$, and $E \leq 1$, where however the firms are able through investment (for instance in advertising) to increase the "variety" coefficient $1-E$. This formulation obtains here, with $E=\delta$, in the special case where each good embodies only one characteristic, different from the one embodied

\footnotetext{
${ }^{10}$ This amounts to say that we analyze an horizontal differentiation problem. Allowing a product to embody more of the two characteristics would lead to study vertical differentiation.

${ }^{11}$ Indeed $\max _{a \in[0,1]}\{a(1-a)\}=\frac{1}{4}$ and $\max (1-\delta)=2$.
} 
in the other product ${ }^{12}$. Of course, $\delta$ is also in this case the coefficient of substitutability between products.

It is interesting to notice that the firms' products are substitutes (resp. complements) in demand whenever $E>0$ (resp. $E<0$ ). Rearranging we obtain

$$
E=\delta+(1-\delta)(a(1-b)+b(1-a))
$$

so that $\delta \geq 0$ is a sufficient condition which ensures that $E \geq 0$. Obviously the case when the two products are substitutes in demand is the most likely. For the firms' products to be complements in demand it is necessary but not sufficient that the characteristics themselves be complementary. The firms must in addition have selected low values of $a$ and $b$, i.e. different enough mixes of the two characteristics. When the firms choose closes mixes of characteristics, their products are substitutes in demand, even when the characteristics are strongly complementary. In Figure 1 below, we have pictured, for the symmetric locations case $(a=b)$, the two areas for which the two products are respectively substitutes and complements in demand.

Figure 1: Product subsituability vs complementarity in the $(a, \delta)$-space

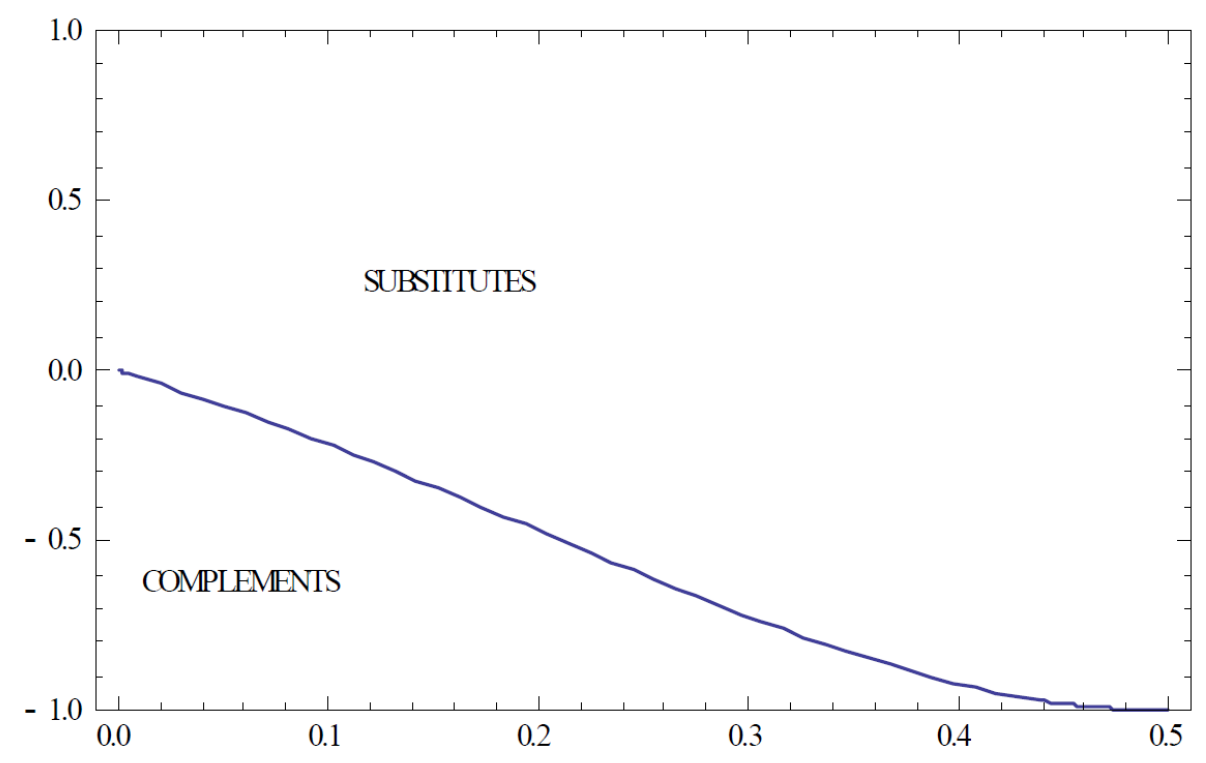

The representative consumer's problem is then to choose the quantities $Q_{1}$ and $Q_{2}$ of the two differentiated goods as well as the quantity $Q_{0}$ of the numeraire good in order to maximize his/her utility, as given by (3) and (2) $)^{13}$, subject to the budget constraint

$$
p_{1} Q_{1}+p_{2} Q_{2}+Q_{0}=R,
$$

and the non-negativity constraint on the consumption of the numeraire good

$$
Q_{0} \geq 0 \Leftrightarrow p_{1} Q_{1}+p_{2} Q_{2} \leq R
$$

where $p_{1}$ and $p_{2}$ are the respective prices of goods 1 and 2 and $R$ is the consumer's

\footnotetext{
${ }^{12}$ That is either $a=b=0$ or $a=b=1$.

${ }^{13}$ i.e. in which we subsitute in (1) for $C_{A}$ and $C_{B}$ their values from (2).
} 
exogenous income. It is generally assumed in the literature that $R$ is large enough to ensure that there is always a strictly positive consumption of the numeraire good, i.e. that the constraint (5) is not binding. It is indeed sensible to discard cases where the consumers would only buy from the two competing firms, i.e. to suppose that the duopolistic sector under study is small with respect to the whole economy. We will however see that this requires $R$ to take arbitrarily large values when $\delta \rightarrow-1$ (perfect complements case) ${ }^{14}$. So we shall make here an assumption which bears simultaneously on $R$ and $\delta$ :

Assumption 1: $R(1+\delta) \geq \frac{4}{9}$.

This assumption will ensure that the constraint (5) never binds in equilibrium whether firms compete in quantities or prices. We complement Assumption 1 by supposing that $R$, while being finite, is very large so that this assumption excludes only the cases where the characteristics are almost pure complements, i.e. a small right neighborhood of -1 .

From this problem, and under this assumption, we easily obtain the following necessary and sufficient conditions:

$$
\begin{aligned}
& p_{1}=1-G Q_{1}-E Q_{2}, \\
& p_{2}=1-D Q_{2}-E Q_{1} .
\end{aligned}
$$

From equation (6), we obtain the direct demand functions:

$$
\begin{aligned}
& Q_{1}=\frac{D\left(1-p_{1}\right)-E\left(1-p_{2}\right)}{D G-E^{2}}, \\
& Q_{2}=\frac{G\left(1-p_{2}\right)-E\left(1-p_{1}\right)}{D G-E^{2}},
\end{aligned}
$$

for the two goods. Notice that the sum $Q_{1}+Q_{2}$ is not constant as it is in the Hotelling model. Our Lancasterian approach allows for a positive elasticity of aggregate demand.

We suppose finally that the two firms have a common constant marginal cost, which, for the sake of simplicity and without any loss of generality, is set equal to zero. The important assumption here is that the composition of the mix of characteristics has no effect on the cost per unit of output. It is made for the sake of simplicity and of symmetry

We now consider a two-stage game in which in a first stage the firms 1 and 2 respectively choose $a$ and $b$, i.e. the mix of the two characteristics of which each unit of their product is made. We assume that these first-stage choices are non cooperative, observable and irreversible. Then, in a second stage, the firms compete in quantities (Cournot equilibrium).

\section{HORIZONTAL DIFFERENTIATION UNDER QUANTITY COMPETITION}

\subsection{Second Stage Cournot Competition}

Given the values of $a$ and $b$ selected in the first stage of the game each firm selects its quantity as a best reply to the quantity of its competitor.

\footnotetext{
${ }^{14}$ This is because, when $a=b=\frac{1}{2}$, the linear-quadratic utility function becomes linear in the limit when $\delta \rightarrow-1$, implying a constant marginal utility $(=1)$ of each good which turns out to be greater than the equilibrium price both in the Cournot case $\left(=\frac{1}{3}\right)$ and the Bertrand one $\left(=\frac{1}{4}\right)$ : the unconstrained demands for the two goods tend then toward infinity.
} 
Assuming that (5) holds, profit maximization yields the following first-order conditions:

$$
1-2 G Q_{1}-E Q_{2}=0
$$

and

$$
1-2 D Q_{2}-E Q_{1}=0
$$

As usual in case of linear demand the quantities are strategic substitutes (resp. complements) whenever the products are substitutes (resp. complements) in demand, i.e. when $E>0$ (resp. $E<0)$..

Straightforwardly the profit function of firm $i$ is strictly concave with respect to $Q_{i}$ and the conditions are not not only necessary but also sufficient. This follows from the fact that $D$ and $G$ are both strictly positive.

The Cournot equilibrium values of the outputs are easily obtained as

$$
Q_{1}^{C}(a, b)=\frac{2 D-E}{4 G D-E^{2}}
$$

and

$$
Q_{2}^{C}(a, b)=\frac{2 G-E}{4 G D-E^{2}}
$$

with the corresponding equilibrium profits

$$
\pi_{1}^{C}(a, b)=G\left[\frac{2 D-E}{4 G D-E^{2}}\right]^{2}
$$

and

$$
\pi_{2}^{C}(a, b)=D\left[\frac{2 G-E}{4 G D-E^{2}}\right]^{2} .
$$

\subsection{Horizontal differentiation}

We are interested only in the pure strategy Nash equilibria of this game which are such that $a \leq 1-b$, i.e. which are such that firm 1 is "at the left" of firm 2 , or, more precisely, such that firm 1's product embodies more of characteristic $A$ and less of characteristic $B$ than firm 2's product. A very simple and striking Proposition can be stated.

PROPOSITION 1 When firms compete in quantities there exists a unique Nash equilibrium in pure strategies $\left(a^{*}, b^{*}\right)$ of the horizontal differentiation game satisfying $a^{*} \leq 1-b^{*}$ which is such that

$$
a^{*}=b^{*}=\frac{1}{2}
$$

Proof: See Appendix A.

Q.E.D.

It is striking that this minimum differentiation result under Cournot competition holds whatever the value of $\delta$. The intuition for this result can be gained by noting that the 
effect of a variation of $a$ on the profit of Firm 1 is the sum of a direct effect, i.e. the partial derivative of $\pi_{1}$ with respect to $a$ for given output levels obtained from (6), plus a strategic effect, which is equal to the partial derivative of $\pi_{1}$ with respect to the rival's output $Q_{2}$ times the derivative of the equilibrium value of $Q_{2}$, as given by (15), with respect to $a$. Let $b=\frac{1}{2}$. The direct effect is

$$
\frac{\partial \pi_{1}\left(Q_{1}^{C}\left(a, \frac{1}{2}\right), Q_{2}^{C}\left(a, \frac{1}{2}\right), a, \frac{1}{2}\right)}{\partial a}=\frac{8(1-2 a)(1-\delta)}{(-7+16(1-a) a(1-\delta)+\delta)^{2}},
$$

which is positive for all $a<\frac{1}{2}$ and negative for all $a>\frac{1}{2}$ : choosing a mix of characteristics closer to the rival's one raises the demand for Firm 1's product. This holds whatever $\delta$. On the other hand, the strategic effect is positive as well since the firms' products are substitutes in demand and, accordingly, strategic substitutes. We indeed check that

$$
\frac{\partial Q_{2}\left(a, \frac{1}{2}\right)}{\partial a}=\frac{16(1-2 a)(1-\delta)}{(-7+16(1-a) a(1-\delta)+\delta)^{2}}
$$

is positive for all $a<\frac{1}{2}$ and negative for all $a>\frac{1}{2}$. Differentiating less one product from the rival's raises the firm's output, pushing its rival down on its reaction function, thus raising the firm's profits. The direct and the strategic effects of choosing closer to the rival's being both positive, this shows that $a=\frac{1}{2}$ is the best reply to $b=\frac{1}{2}$.

Notice that, while these results look familiar, they imply that the strategic product design game may exhibit a "prisoner dilemma" property. Indeed, when the characteristics are independent (i.e. $\delta=0)$, by coordinating on the pair $(a=0, b=0)$ the two firms would specialize each in a different characteristic and thereby become monopolists. This is not however an equilibrium of the non-cooperative strategic product design game. At $(a=0, b=0)$ an increase of $a$ has indeed both a positive direct effect (at the corresponding equilibrium quantities, $\left.\frac{\partial \pi_{1}\left(Q_{1}^{C}(0,0), Q_{2}^{C}(0,0), 0,0\right)}{\partial a}=\frac{1}{4}\right)$ and a positive strategic effect since the equilibrium rival's output decreases, $\left.\frac{\partial Q_{2}(0,0)}{\partial a}=-\frac{1}{4}\right)$.

Obviously, this results sounds familiar. However, it is obtained in a model where the firms may choose in a first stage whether they will offer complements or substitutes and not only the degree to which they are substitutes, as is the case in the Hotelling's model.

COROLlary 1 At the unique Cournot-Nash Equilibrium of the two-stage game the firms outputs, prices and profits are decreasing in the coefficient $\delta$ of substitutability between the product characteristics, i.e. for $i=1,2$ :

$$
\begin{aligned}
Q_{i}^{*} & =\frac{2}{3(1+\delta)}, \\
p_{i}^{*} & =\frac{1}{3}, \\
\pi_{i}^{*} & =\frac{2}{9(1+\delta)} .
\end{aligned}
$$

It is interesting to notice that the equilibrium prices are constant, whatever the degree of substitutability $\delta$ between the characteristics, while the equilbrium outputs and profits decrease in $\delta$. It is interesting to determine what in the variation (decrease) of equilibrium profits $\pi_{i}^{*}$ with respect to $\delta$ is to be attributed to a direct effect on the demand for good $i$ 
and to a strategic effect. We define here the direct effect as the effect of a variation of $\delta$ for fixed (equilibrium) values of the mixes of characteristics $a^{*}$ and $b^{*}$ and of the output levels $Q_{1}^{*}$ and $Q_{2}^{*}$ and the strategic effect as the effect resulting from induced changes of the equilibrium values of $a^{*}, b^{*}, Q_{1}^{*}$ and $Q_{2}^{* 15}$.

For this purpose and given $a^{*}=b^{*}=\frac{1}{2}$, Firm i's equilibrium profits may be written as

$$
\pi_{i}^{*}(\delta)=Q_{i}^{*}(\delta)\left(1-\frac{1}{2}(1+\delta)\left(Q_{i}^{*}(\delta)+Q_{j}^{*}(\delta)\right)\right)
$$

so that, using he Envelope Theorem,

$$
\begin{aligned}
\frac{d \pi_{i}^{*}(\delta)}{d \delta} & =-\frac{1}{2}(1+\delta) Q_{i}^{*}(\delta) \frac{d Q_{j}^{*}(\delta)}{d \delta}-\frac{1}{2} Q_{i}^{*}(\delta)\left(Q_{i}^{*}(\delta)+Q_{j}^{*}(\delta)\right) \\
& =\frac{2}{9(1+\delta)^{2}}-\frac{4}{9(1+\delta)^{2}}=-\frac{2}{9(1+\delta)^{2}}<0 .
\end{aligned}
$$

The first term in the RHS of the above equation is the (positive) strategic effect of an increase of $\delta$, effect which comes from the reduction of the competitor equilibrium output level, while the second is the direct (negative) effect which comes from the reduction of the demand, other thing equal ${ }^{16}$. The direct effect unambiguously dominates the strategic one.

Given Assumption 1, the positivity constraint (5) holds in equilibrium since

$$
\sum_{i=1}^{2} \pi_{i}^{*}=\frac{4}{9(1+\delta)} \leq R .
$$

\section{HORIZONTAL DIFFERENTIATION UNDER PRICE COMPETITION}

\subsection{Second-Stage Bertrand Competition}

Disregarding non-negativity constraints in the firms' optimization problems leads to the following Bertrand equilibrium prices:

$$
\begin{aligned}
& p_{1}=\frac{2 D G-E^{2}-E G}{4 D G-E^{2}}, \\
& p_{2}=\frac{2 D G-E^{2}-E D}{4 D G-E^{2}}
\end{aligned}
$$

These values are both zero whenever $1-a-b=0$, i.e. when the two firms offer exactly the same products. More interestingly they are negative for some sets of parameter values. In Figure 2 we have plotted the candidate value for $p_{1}$ as a function of $b$ for $\delta=0$

\footnotetext{
${ }^{15}$ Notice that this definition is the more natural but is not obvious. We could instead have focused on the equilibrium payoffs at stage 1 of the game as functions of the values of $a^{*}(\delta), b^{*}(\delta)$ and $\delta$, accounting for the fact that the equilibrium, second-stage, output levels are themselves functions of $a^{*}(\delta), b^{*}(\delta)$ and $\delta$. Since the equilibrium mixes are constant, this would have led to the paradoxical conclusion of the absence of any strategic effect.

${ }^{16}$ When the characteristics which they embody become closer substitutes, the goods become less desirable from the consumers' point of view, i.e. their marginal utility decreases at any given quantities.
} 
and $a=0.2$.

Figure 2: Price as function of "location"

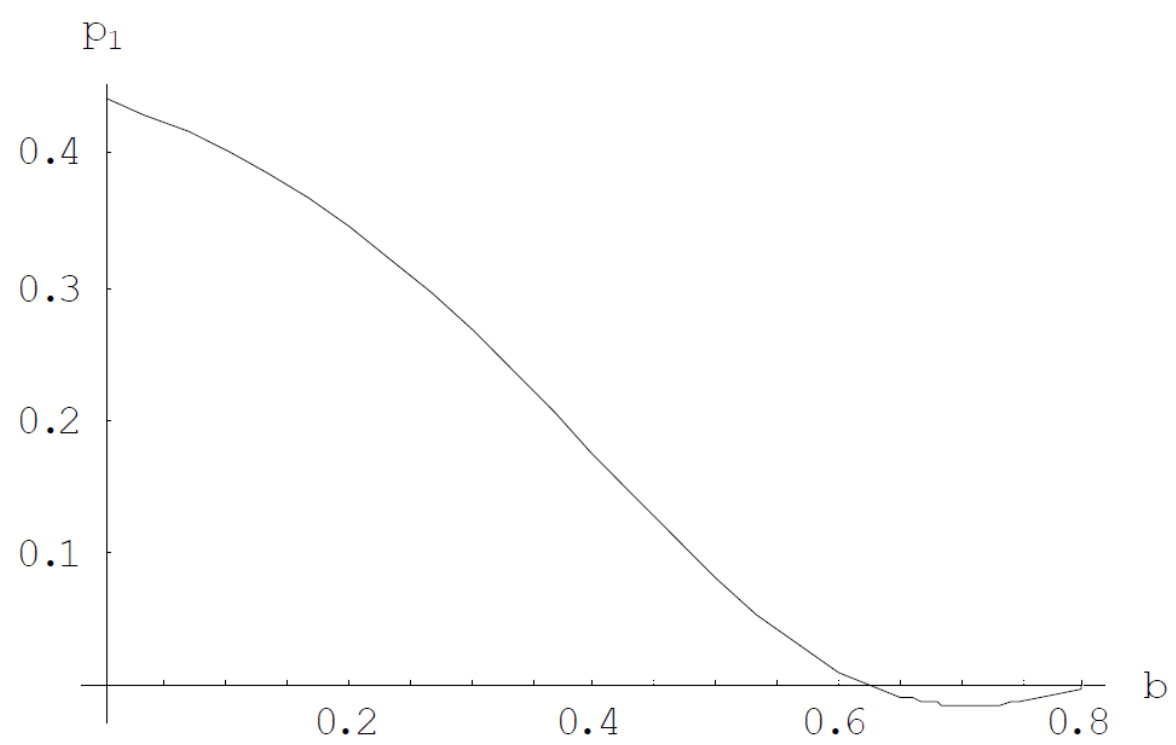

More generally when Firm 2 moves "leftward" toward a Firm 1 "located" in the first half of the interval $[0,1]$, its price $p_{1}$ goes down due to increased competition (a negative "strategic effect") and becomes equal to zero when Firm 2 becomes to propose a product mix close but not identical to Firm 1's. Notice that $p_{2}$ remains always positive as long as $1-a-b>0$.

Proposition 2 When $1-a-b \geq 0$ the Nash equilibrium $\left(p_{1}^{*}, p_{2}^{*}\right)$ is such that:

(i) when $b \leq \frac{2-3 a+(1-\delta) 2 a^{2}+\delta(1+a)}{3-4 a+(1-\delta) 4 a^{2}+\delta(1+4 a)}$ and $a \leq \frac{2-3 b+(1-\delta) 2 b^{2}+\delta(1+b)}{3-4 b+(1-\delta) 4 b^{2}+\delta(1+4 b)} \quad p_{1}^{*}$ and $p_{2}^{*}$ are given by (14).

(ii) when $1-a \geq b \geq \frac{2-3 a+(1-\delta) 2 a^{2}+\delta(1+a)}{3-4 a+(1-\delta) 4 a^{2}+\delta(1+4 a)} 17$

$$
\begin{aligned}
& p_{1}^{*}=0 \\
& p_{2}^{*}=\frac{(1-2 a)(1-a-b)(1-\delta)}{2(1-2 a(1-\delta)(1-a))}
\end{aligned}
$$

(iii) when $1-b \geq a \geq \frac{2-3 b+(1-\delta) 2 b^{2}+\delta(1+b)}{3-4 b+(1-\delta) 4 b^{2}+\delta(1+4 b)} 18$

$$
\begin{aligned}
& p_{1}^{*}=\frac{(1-2 b)(1-a-b)(1-\delta)}{2(1-2 b(1-\delta)(1-b))} \\
& p_{2}^{*}=0
\end{aligned}
$$

PROOF: see Appendix B.

Q.E.D.

The equilibrium prices are both strictly positive for all $a=b \leq \frac{1}{2}$.

We generalize in Appendix this Proposition in order to cover the mirror case when $1-a-b \leq 0$. Corresponding equilibrium profits are easily derived in each case: the

\footnotetext{
${ }^{17}$ It should be noticed that this implies that $a \leq \frac{1}{2}$.

${ }^{18}$ nitice that this implies that $b \leq \frac{1}{2}$.
} 
profits of Firm 2 do not depend on its mix $b$ as long as $p_{1}^{*}=0$. The same holds for the profits of Firm 1 when $p_{2}^{*}=0$.

\subsection{First-Stage Product Selection}

In the first stage each firm chooses its product mix. Proposition 3 below shows that the result hinges crucially on the value of $\delta$, i.e. on the degree of substitutability/complementarity between the two characteristics with the opposite outcomes of maximum and minimum differentiation as extreme cases corresponding to specific values of $\delta$.

PROPOSITION 3 There are two and only two mirror subgame-perfect Nash equilibria of the two-stage game which are $a^{*}=b^{*}=\max \left\{0, \frac{1}{2}-\frac{\sqrt{1-\delta^{2}}}{(1-\delta) 2 \sqrt{5}}\right\}$ and $a^{*}=b^{*}=$ $\min \left\{1, \frac{1}{2}-\frac{\sqrt{1-\delta^{2}}}{(1-\delta) 2 \sqrt{5}}\right\}$.

Proof: see Appendix C.

Q.E.D.

The two equilibria are mirror images of each other. Our results are pictured below in Figure 3 for the case where Firm 1 is the firm "located" at the left.

Figure 3: a (b) as a function of $\delta$

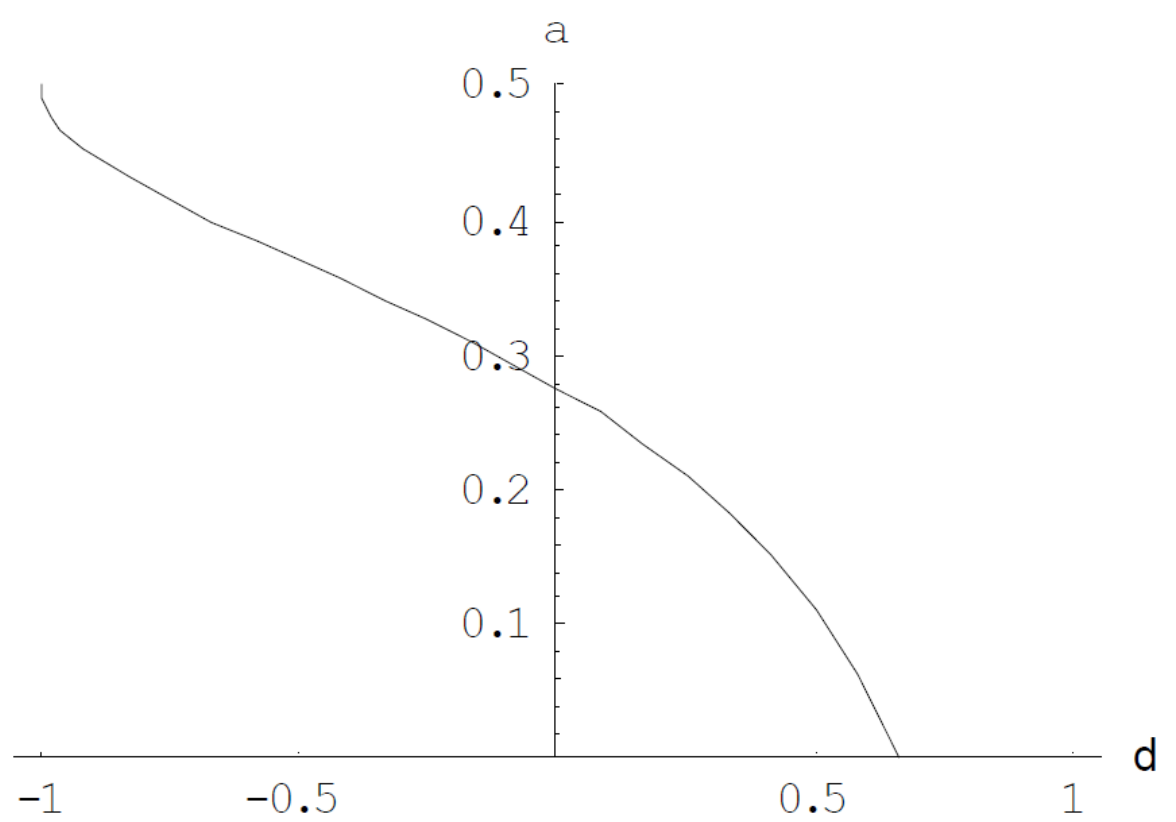

The two forces which interplay to determine the equilibrium product mixes are, as usual, a (generally positive) direct effect (a change in a firm's product mix changes the demand for its product at constant prices) and a (generally negative) strategic effect (the same change in the product mix leads to a change in the rival's equilibrium price). For the sake of illustration, let us consider the case of independent characteristics and let $b=0$. The direct effect is, from Proposition 2,

$$
\begin{aligned}
& \frac{\partial \pi_{1}^{B}\left(p_{1}^{*}(a, 0), p_{2}^{*}(a, 0)\right)}{\partial a}=\frac{(2-3 a)\left(2-3 a+2 a^{2}\right)}{(1-a)\left(-4+8 a-7 a^{2}\right)^{2}}, \text { if } a<\frac{2}{3}, \\
& \frac{\partial \pi_{1}^{B}\left(p_{1}^{*}(a, 0), p_{2}^{*}(a, 0)\right)}{\partial a}=0, \text { if } a \geq \frac{2}{3} .
\end{aligned}
$$


It is positive for all $a<\frac{2}{3}$. Getting closer to the rival's mix raises the demand for the firm's product. Now, to account for the strategic effect, we have from Proposition 2

$$
\begin{aligned}
& \frac{\partial p_{2}^{*}(a, 0)}{\partial a}=\frac{11 a^{2}-4(1+a)}{\left(-4+8 a-7 a^{2}\right)^{2}}<0, \text { if } a<\frac{2}{3}, \\
& \frac{\partial p_{2}^{*}(a, 0)}{\partial a}=0, \text { if } a \geq \frac{2}{3} .
\end{aligned}
$$

Since in this range the products are substitutes, the strategic effect of an increase in $a$ is negative: choosing a product mix closer to the rival leads to a fiercer competition detrimental to the firm's profits. As usual in the case of price competition, the direct and strategic effects push in opposite directions ${ }^{19}$.

Equilibrium maximal differentiation ( $a^{*}=b^{*}=0$ ) obtains if and only if $\delta \geq \frac{2}{3}$, i.e. if the characteristics are close substitutes. If, on the contrary, $\delta \rightarrow-1$, we tend toward minimum differentiation, i.e. $a^{*}=b^{*} \rightarrow \frac{1}{2}$. The intuition for this result is that, in this case, the direct effect of an increase in $a$ on Firm 1's profits, that is the effect on demand for product 1 at given prices computed for $b$ close to $\frac{1}{2}$, becomes very large: the consumers dislike comparatively very much a product which does not embody equally the two characteristics. This direct negative effect of product differentiation becoming stronger with respect to the positive strategic (competition softenig) effect, the firms differentiate less their products.

Notice that a prisoner's dilemma appears also in this case when $\delta=0$. Coordinating on maximal differentiation $(a=0, b=0)$ would allow each firm to be the monopolist seller of the only characteristic embodied in its product. However, as shown above, maximal differentiation is not a non-cooperative equilibrium of the product design game when $\delta=0$, the best reply to $b=0$ being $a=0.4$ rather than $a=0$.

Now, when $\delta$ increases from -1 to $\frac{2}{3}$, the two firms differentiate more and more their product mixes, thus succeeding in maintaining constant equilibrium prices (see Corollary below) but demands decrease since products which embody more similar characteristics become less attractive, so that equilibrium profits decrease. Notice that greater differentiation has a negative direct effect (at constant prices, the demand to the firm is the lower, the more it differentiates its product from the rival's) and a positive strategic effect (competition dampening leading to a higher rival's equilibrium price). The intuition behind the increasing differentiation result in this model is that the negative direct effect on demand of greater differentiation is the smaller the more the characteristics are substitutable: in the limit, when $\delta=1$, the characteristics become perfect substitutes, the direct effect vanishes and there remains only the positive strategic effect.

\section{COROLlary 2 At the Bertrand-Nash equilibria of the two-stage game,}

\footnotetext{
${ }^{19}$ It is easy to show that the former (resp. the latter dominates when $a<0.4$ (resp. $a>0.4$ ), i.e. $a=0.4$ is the best reply to $b=0$.
} 


$$
\begin{aligned}
p_{i}^{*} & =\frac{1}{4}, \forall \delta \in\left(-1, \frac{2}{3}\right] ; \\
p_{i}^{*} & =\frac{2-\delta^{2}-\delta}{4-\delta^{2}}, \forall \delta \in\left[\frac{2}{3}, 1\right] ; \\
\pi_{i}^{*} & =\frac{3}{16(1+\delta)}, \forall \delta \in\left(-1, \frac{2}{3}\right] ; \\
\pi_{i}^{*} & =\frac{1-\delta}{(2-\delta)^{2}(1+\delta)} ; \forall \delta \in\left[\frac{2}{3}, 1\right] .
\end{aligned}
$$

For any given $(a, b), \delta$ measures the degree of competition between the firms. The more substitutable $A$ and $B$, the harsher the price competition and the stronger the incentive to differentiate the products. The firms thus succeed to maintain constant equilibrium prices (equal to $\frac{1}{4}$ ) as long as $\delta \leq \frac{2}{3}$. However the demands for the two goods decrease when the characteristics become closer substitutes so that, despite the greater product differentiation, the equilibrium profits of the firms fall when $\delta$ increases.

It is interesting to identify precisely the strategic and direct effects of an increase of $\delta$, using mutatis mutandis the same definition as in the previous Section.

Let us consider the simplest case $\delta \geq \frac{2}{3}$ in which the equilibrium mixes of characteristics are constant, i.e. $a^{*}=b^{*}=0$. Here the strategic effect of a variation of $\delta$ on firm $i$ 's profits is the induced change into the competitor's price $p_{j}^{*}(\delta)$ occurring in the second stage of the game for the fixed equilibrium mixes. Firm i's profits can be written as

$$
\pi_{i}^{*}(\delta)=p_{i}^{*}(\delta) \frac{\left(1-p_{i}^{*}(\delta)\right)-\delta\left(1-p_{j}^{*}(\delta)\right)}{1-\delta^{2}},
$$

so that, using the Envelope Theorem,

$$
\begin{aligned}
\frac{d \pi_{i}^{*}(\delta)}{d \delta} & =-p_{i}^{*}(\delta) \frac{\delta}{1-\delta^{2}} \frac{1}{(2-\delta)^{2}}+p_{i}^{*}(\delta) \frac{-\left(1-\delta^{2}\right)-2 \delta p_{i}^{*}(\delta)+\left(1+\delta^{2}\right) p_{j}^{*}(\delta)}{\left(1-\delta^{2}\right)^{2}} \\
& =-\frac{\delta}{(2-\delta)^{3}(1+\delta)}-\frac{1-\delta}{\left(2+\delta-\delta^{2}\right)^{2}}<0 .
\end{aligned}
$$

The first term in the RHS of the above equation is the (negative) strategic effect of an increase of $\delta:$ the greater the degree of substitutability between the characteristics, the smaller the competitor's equilibrium price and so the smaller $i$ 's profits. The second term is the (negative) direct effect of an increase of $\delta$ : at given prices, such an increase reduces the demand for good $i$ since it makes both goods less attractive to consumers. So both effects are negative, contrary to the Cournot competition case.

When $\delta<\frac{2}{3}$, equilibrium mixes change with $\delta$ while equilibrium prices are constant. Since $a^{*}(\delta)=b^{*}(\delta)$, when $\delta$ increases the firms change their equilibrium mixes in opposite directions by the same amounts. The effects of these changes on firms' demands and profits are exactly offsetting. Indeed, for instance firm 1's profits write as

$$
\pi_{1}^{*}\left(\delta, a^{*}(\delta), b^{*}(\delta)\right)=\frac{1}{4}\left(\frac{\frac{3}{4}(D-E)}{D G-E^{2}}\right)
$$

where $D=G$ and $E$ are known functions of $a, b$ and $\delta$. Given that $a^{*}(\delta)=b^{*}(\delta)$, the strategic effect is simply 


$$
\begin{aligned}
& {\left[\frac{\partial \pi_{1}^{*}\left(\delta, a^{*}(\delta), b^{*}(\delta)\right)}{\partial a}+\frac{\partial \pi_{1}^{*}\left(\delta, a^{*}(\delta), b^{*}(\delta)\right)}{\partial b}\right] a^{*^{\prime}}(\delta) } \\
= & {\left[\frac{6}{16} \frac{a^{*}(\delta)-b^{*}(\delta)}{\left(1-a^{*}(\delta)-a^{*}(\delta)\right)^{2}(1+\delta)}\right] a^{*^{\prime}}(\delta)=0 . }
\end{aligned}
$$

When $\delta<\frac{2}{3}$, all the negative effect of an increase of $\delta$ on firms' equilibrium profits come from its direct negative effect on the demands for the two goods.

Finally, notice that Assumption 1 ensures that the positivity constraint (5) holds in equilibrium since

$$
\sum_{i=1}^{2} \pi_{i}^{*} \leq \frac{3}{8(1+\delta)}<\frac{4}{9(1+\delta)} \leq R .
$$

\section{WELFARE ANALYSIS AND COMPARISONS}

It is illuminating to characterize the first-best optimum, i.e. the output levels and product mixes which maximize the aggregate social surplus

$$
W=Q_{1}+Q_{2}-\frac{1}{2}\left(G Q_{1}^{2}+D Q_{2}^{2}\right)-E Q_{1} Q_{2}+R
$$

Notice that, given that marginal costs have been normalized to zero, $W$ corresponds simply to consumer's utility which is always maximized when he/she consumes equal amounts of the two characteristics at zero prices (equal to marginal costs). In turn, consuming equal amounts of the characteristics is possible iff the product mixes allow for that, i.e. if both mixes do not embody a strictly greater amount of one of the characteristics. So the social optimum is defined by equating the marginal utilities of the two goods to their marginal costs of production, here normalized to 0 , so that

$$
\begin{aligned}
Q_{1}^{O p t} & =\frac{D-E}{D G-E^{2}}=\max \left\{\frac{1-2 b}{(1-a-b)(1+\delta)}, 0\right\}, \\
Q_{2}^{O p t} & =\frac{G-E}{D G-E^{2}}=\max \left\{\frac{1-2 a}{(1-a-b)(1+\delta)}, 0\right\},
\end{aligned}
$$

and by stipulating that the $a^{O p t}$ and $b^{O p t}$ should not be such that $a^{O p t}<\frac{1}{2}<b^{O p t}$ nor such that $b^{O p t}<\frac{1}{2}<a^{O p t}$. Clearly there is a continuum of values of $a$ and $b$ and $Q_{1}$ and $Q_{2}$ which satisfy these conditions. Nevertheless, at optimum we should always observe that

$$
C_{X}^{O p t}=\frac{1}{1+\delta}=W^{O p t}, X=A, B .
$$

This simple analysis teaches us that the exact composition of product mixes is not an issue for welfare analysis in so far as they may allow consumers to adjust their purchases of the two goods in order to reach their desired aggregate characteristics mix ${ }^{20}$. And this is what indeed happens both at the Cournot and the Bertrand equilibria at which it is never observed that $a^{*}<\frac{1}{2}<b^{*}$ nor that $b^{*}<\frac{1}{2}<a^{*}$. The only source of inefficiency

\footnotetext{
${ }^{20}$ i.e. excess or insufficient horizontal product differentiation is not an issue.
} 
is the usual, imperfect competition, one, namely that equilibrium prices are in both cases above marginal costs. Cournot equilibrium appears in our framework, contrary to Brander and Spencer (2015), always more inefficient than Bertrand equilibrium despite the fact that Bertrand duopolists differentiate more than products than their Cournot counterparts. More precisely one obtains

$$
W^{\text {Cournot }}=\frac{8}{9} \frac{1}{1+\delta}=\frac{8}{9} W^{O p t}
$$

and

$$
\begin{aligned}
W^{\text {Bertrand }} & =\frac{3-2 \delta}{(\delta+1)(\delta-2)^{2}}, \forall \delta \in\left[\frac{2}{3}, 1\right], \\
& =\frac{15}{16} \frac{1}{1+\delta}, \forall \delta \in\left(-1, \frac{2}{3}\right] .
\end{aligned}
$$

It follows that for all $\delta \in(-1,1), W^{\text {Cournot }}<W^{\text {Bertrand }}<W^{\text {Opt }}$. Only for $\delta=1$, i.e. the perfect substitutes case ${ }^{21}, W^{B e r t r a n d}=W^{O p t}$.

\section{CONCLUDING REMARKS}

Besides confirming in a new framework the fact that Cournot competition entails minimal differentiation, the present model has shown that the degree of substitutability / complementary between the characteristics of the products is a main determinant of the equilibrium horizontal product differentiation when firms compete in prices. Under Bertrand competition indeed, maximal differentiation occurs iff the degree of substitutability between characteristics is above a critical value (equal to 2/3) Below this value, firms do not differentiate maximally and the extent of differentiation decreases when the degree of substitutability between characteristics decreases (or the degree of complementarity increases) This is because a change in the respective proportions of more complementary characteristics has a greater impact on the demand for the product, i.e. the direct effect of horizontal product differentiation choices is then greater.

These results have obtained using a specific, linear-quadratic utility functions. As they look quite intuitive, we are rather confident that they are robust. The minimal differentiation result under Cournot competition, on one hand, comes from the negative strategic effect of increasing differentiation which is itself the consequence of strategic substitutability of outputs, a feature which would obtain for a very large class of utility functions. On the other hand, under Bertrand competition, the key of our results is that the direct negative effect of greater differentiation is stronger the more complementary are the characteristics, what is a rather intuitive property.

A first natural extension would be consider consumers with different tastes for characteristics. It would be for instance interesting to investigate the strategic consequences of the empirical results by Gracia, Barreiro-Hurlé, and Galán (2014) showing that two characteristics may be complements for a given group of consumers but substitutes for another one. Another interesting extension would be to incorporate Johnson and Myatt (2006) insights about the choice of the optimal mix of characteristics into our strategic

\footnotetext{
${ }^{21}$ Remember that since each product embodies one and only one (different) characteristic, the perfect substitutability between characteristics entails a perfect substitutability between products.
} 


\section{framework.}

A second extension would be to consider more than two characteristics, in order to see whether this entails in an Lancasterian model the same qualitative changes as have been put into light by Irmen, Thisse, et al. (1998) in a spatial differentiation framework.

The present model also may be extended to deal with vertical as well as with horizontal differentiation. We have considered above the problem of determining the equilibrium mix between the characteristics, given that the unit cost of the product remains unchanged when this mix is modified. A more general formulation would allow for considering products with more of the two characteristics, of course at a higher unit cost (for instance by considering marginal costs of characteristics). Of course consumers would prefer at the same price a product with more of the two characteristics, i.e. this would allow for vertical differentiation.

\section{APPENDIX A: PROOF OF PROPOSITION 1}

(i) Differentiating (12) with respect to $a$ for $b=\frac{1}{2}$ we obtain the first-order condition

$$
\frac{\partial \pi_{1}^{C}\left(a, \frac{1}{2}\right)}{\partial a}=\frac{8(1-2 a)(1-\delta)(-9-\delta+16 a(1-a)(1-\delta))}{(-7+16 a(1-a)(1-\delta)+\delta)^{3}}=0
$$

Since $(-9-\delta+16 a(1-a)(1-\delta))<0$ for all $(a, \delta) \in[0,1] \times(-1,1)$ this equation has only one real root which is $a=\frac{1}{2}$. It is moreover easy to show that $\frac{\partial^{2} \pi_{1}^{C}}{\partial a^{2}}\left(\frac{1}{2}, \frac{1}{2}\right)=-\frac{80(1-\delta)}{27(1+\delta)^{2}}<0$, i.e. that $\frac{1}{2}=\operatorname{ArgMax}$ $\partial \pi_{1}^{C}\left(a, \frac{1}{2}\right)$. By a similar argument it is possible to show that $\frac{1}{2}=\underset{b}{\operatorname{ArgMax}} \partial \pi_{2}^{C}\left(\frac{1}{2}, b\right)$.

(ii) To prove that $\left(\frac{1}{2}, \frac{1}{2}\right)$ is the only equilibrium let us first evaluate $\frac{\partial \pi_{1}^{C}(a, b)}{\partial a}$ at $a=b$. We obtain

$$
\frac{\partial \pi_{1}^{C}(b, b)}{\partial a}=(1-2 b) H(b, \delta)
$$

where $H(b, \delta)=\frac{2(1-\delta)\left(2+8 b^{3}(2-b)(1-\delta)^{2}+\delta(1+\delta)-2 b\left(3-4 \delta+\delta^{2}\right)+2 b^{2}\left(7-12 \delta+5 \delta^{2}\right)\right)}{2-6 b(1-b)(1-\delta)-\delta}$. It is easy to see that $H(b, \delta)>$ 0 since, for all $\delta \in(-1,1)$, (a) the equation $H(b, \delta)=0$ has no real $\operatorname{root}^{22}$ and (b) $H(0, \delta)=2(1-$ $\delta) \frac{2+\delta+\delta^{2}}{(2-\delta)(2+\delta)^{3}}>0$

Let us now consider generally how $\pi_{1}^{C}(a, b)$ varies with $a$. Using Mathematica it is possible to show that $\pi_{1}^{C}(a, b)$ tends, respectively by increasing and by decreasing values, toward the same positive limit as $a$ tends toward $+\infty$ and as it tends toward $-\infty$. Moreover the equation $\frac{\partial \pi_{1}^{C}(a, b)}{\partial a}=0$ has at most two real roots (and only one when $b=\frac{1}{2}$ ), the first which corresponds to a maximum and a second to a minimum. This is pictured below in the case where $\delta=0$ and $b=0$.

It follows that (a) the best reply correspondence of firm 1, i.e. $R_{1}(b)=\underset{a}{\operatorname{Arg}} \underset{a x}{ } \pi_{1}^{C}(a, b)$ is single valued and (b) since $\operatorname{sign}\left\{\frac{\partial \pi_{1}^{C}(b, b)}{\partial a}\right\}=\operatorname{sign}\{1-2 b\}, R_{1}(b)>(<) b \Longleftrightarrow b<(>) \frac{1}{2}$. By the same argument we would show that $R_{2}(a)>(<) a \Longleftrightarrow a<(>) \frac{1}{2}$ where $R_{2}(a)$ is the best-reply function of firm 2 . This is sufficient to ensure that the two best reply functions can cut only once at $\left(\frac{1}{2}, \frac{1}{2}\right)$.

\section{APPENDIX B: GENERALIZATION OF PROPOSITION 2}

For proving Proposition 3 we need to generalize Proposition 2 to the case where $1-a-b \leq 0$. In Figure 5 above Case (i) corresponds to the mirror areas A, case (ii) to the mirror areas $\mathrm{C}$ and case (iii) to the mirror areas $\mathrm{B}$ and, of course, the set of couples $(a, b)$ such that $1-a-b \leq 0$ is above the line $b=1-a$. Algebraically when $1-a-b \leq 0 \quad$ Case (i) occurs when $b \geq \frac{2-3 a+(1-\delta) 2 a^{2}+\delta(1+a)}{3-4 a+(1-\delta) 4 a^{2}+\delta(1+4 a)}$ and $a \geq \frac{2-3 b+(1-\delta) 2 b^{2}+\delta(1+b)}{3-4 b+(1-\delta) 4 b^{2}+\delta(1+4 b)}$, Case (ii) when $1-a \leq b \leq \frac{2-3 a+(1-\delta) 2 a^{2}+\delta(1+a)}{3-4 a+(1-\delta) 4 a^{2}+\delta(1+4 a)}$ and Case (iii) to

\footnotetext{
${ }^{22}$ This can be checked using Mathematica.
} 
Strategic product design under duopoly

Figure 4: $\pi_{1}^{C}(a ; 0)$ as function of $a(\delta=0)$

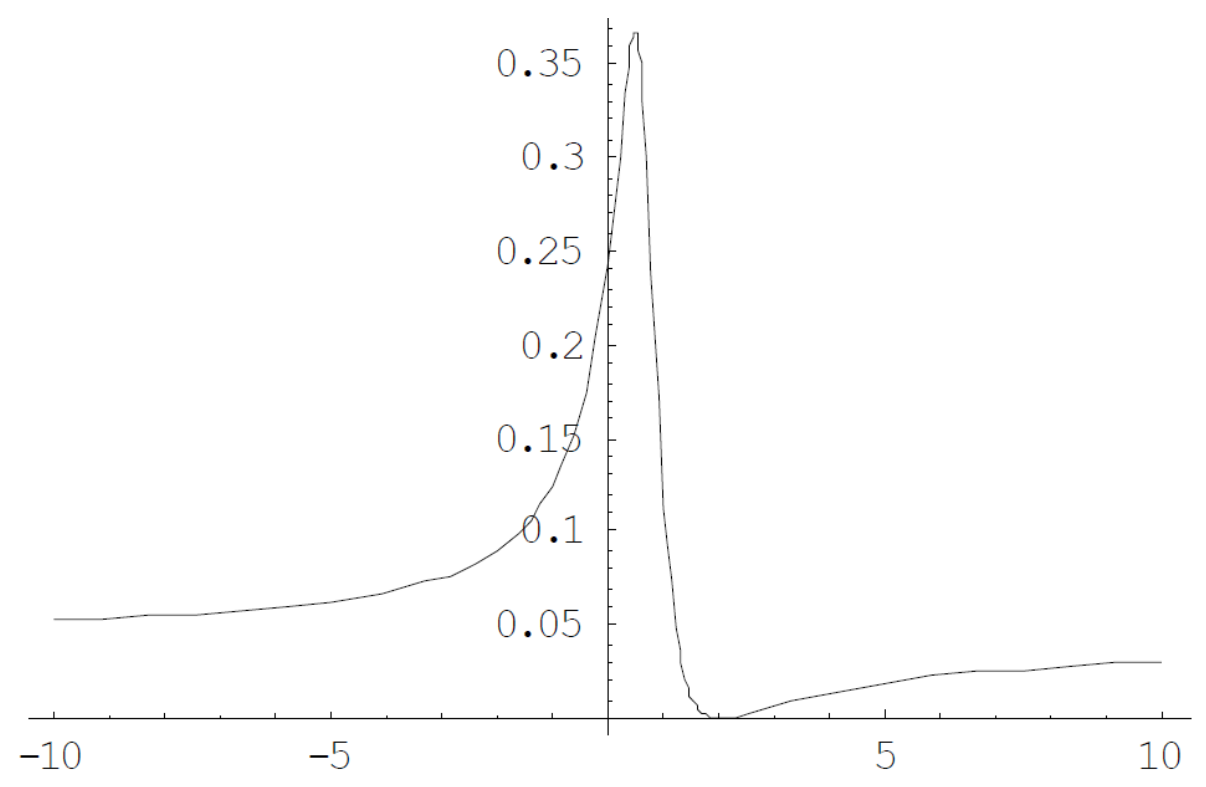

Figure 5: The possible cases in the $a-b$ space

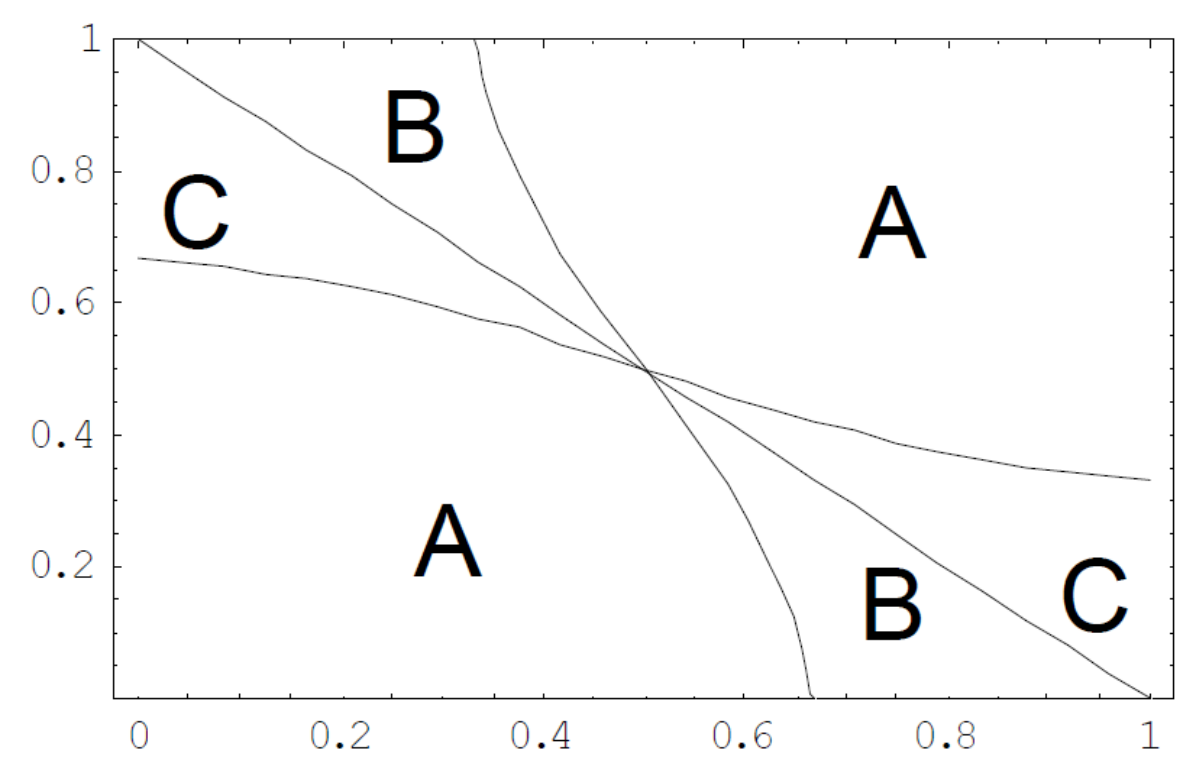


$1-b \leq a \leq \frac{2-3 b+(1-\delta) 2 b^{2}+\delta(1+b)}{3-4 b+(1-\delta) 4 b^{2}+\delta(1+4 b)}$.

\section{APPENDIX C: PROOF OF PROPOSITION 3}

(i) In the case of positive prices (Case (i) of Proposition 2, area $A$ of Figure 5) differentiating $\pi_{1}$ with respect to $a$ and $\pi_{2}$ with respect to $b$ we obtain a system of two equations in $a$ and $b$ which are too long to be reproduced here ${ }^{23}$. This system has only three real solutions which are $\left(\frac{1}{2}, \frac{1}{2}\right),\left(\frac{1}{2}-\frac{\sqrt{1-\delta^{2}}}{(1-\delta) 2 \sqrt{5}}, \frac{1}{2}-\frac{\sqrt{1-\delta^{2}}}{(1-\delta) 2 \sqrt{5}}\right)$ and $\left(\frac{1}{2}+\frac{\sqrt{1-\delta^{2}}}{(1-\delta) 2 \sqrt{5}}, \frac{1}{2}+\frac{\sqrt{1-\delta^{2}}}{(1-\delta) 2 \sqrt{5}}\right)$ and which all satisfy the non negative equilibrium prices requirement (i.e. belong to $A$ ). The solution $\left(\frac{1}{2}, \frac{1}{2}\right)$ does not correspond to an equilibrium since the second-order derivatives respectively of $\pi_{1}$ with respect to $a$ and of $\pi_{2}$ with respect to $b$ evaluated at $\left(\frac{1}{2}, \frac{1}{2}\right)$ are both equal to $\frac{16(1-\delta)}{9(1+\delta)^{2}}>0$.

(ii) Let us show that $a^{*}=b^{*}=\max \left\{0, \frac{1}{2}-\frac{\sqrt{1-\delta^{2}}}{(1-\delta) 2 \sqrt{5}}\right\}$ is indeed an equilibrium.

Suppose first that $\delta \leq \frac{2}{3}$ and study the properties of $\pi_{1}\left(a, \frac{1}{2}-\frac{\sqrt{1-\delta^{2}}}{(1-\delta) 2 \sqrt{5}}\right)$ as a function of $a$. It is first increasing as $a$ increases from 0 , has a local maximum at $a=\frac{1}{2}-\frac{\sqrt{1-\delta^{2}}}{(1-\delta) 2 \sqrt{5}} 24$ (where $\frac{\partial^{2} \pi_{1}}{\partial a^{2}}=-\frac{225(1-\delta)}{128(1+\delta)^{2}}<$ $0)$ and is then decreasing with $a$ until $a=\frac{1}{2}-\frac{\sqrt{5\left(1-\delta^{2}\right)}}{22(1-\delta)}$ where one reaches the area B corresponding to case (iii) of Proposition 2. Straightforwardly in this area it continues to decrease when $a$ increases. Finally with further increases of $a$ (larger than $1-b$ ) one reaches the area $\mathrm{C}$ corresponding to Case (ii) of Proposition 2 in which $\pi_{1}=0$. Notice that the inequality $b \leq \frac{2-3 a+(1-\delta) 2 a^{2}+\delta(1+a)}{3-4 a+(1-\delta) 4 a^{2}+\delta(1+4 a)}$ continues to hold true until $a=1^{25}$. This is sufficient to prove that $a=\frac{1}{2}-\frac{\sqrt{1-\delta^{2}}}{(1-\delta) 2 \sqrt{5}}$ is the unique global maximum of $\pi_{1}\left(a, \frac{1}{2}-\frac{\sqrt{1-\delta^{2}}}{(1-\delta) 2 \sqrt{5}}\right)$ with respect to $a$. Of course the same argument shows that $b=\frac{1}{2}-\frac{\sqrt{1-\delta^{2}}}{(1-\delta) 2 \sqrt{5}}$ is the unique global maximum of $\pi_{2}\left(\frac{1}{2}-\frac{\sqrt{1-\delta^{2}}}{(1-\delta) 2 \sqrt{5}}, b\right)$ with respect to $b$.

Suppose then that $\delta \geq \frac{2}{3}$. a similar argument is enough to show that $a=0$ is the unique global maximum of $\pi_{1}(a, 0)$ as a function of $a$.

A similar argument would show that $a^{*}=b^{*}=\min \left\{1, \frac{1}{2}+\frac{\sqrt{1-\delta^{2}}}{(1-\delta) 2 \sqrt{5}}\right\}$ is an equilibrium as well.

(iii) It remains only to show that there is no equilibrium such that $\left(a^{*}, b^{*}\right) \notin A$. Suppose for instance without any loss of generality that $\left(a^{*}, b^{*}\right) \in B$. Clearly in this case $\pi_{1}\left(a^{*}, b^{*}\right)=0$. From Figure 5 it is straightforward that Firm 1 could select a value of $a$ so as to move to areas $B$ or $C$ and earn strictly positive profits $^{26}$. It follows that $a^{*}$ cannot be a best reply to $b^{*}$.

\footnotetext{
${ }^{23}$ The Mathematica file is available on demand.

${ }^{24}$ As defined by (10) $\frac{\partial \pi_{1}}{\partial a}=0$ has two real roots, the second and largest one being equal to $\frac{1}{2}+\frac{5 \sqrt{5\left(1-\delta^{2}\right)}}{14(1-\delta)}$ which would correspond to a local minimum (since $\frac{\partial^{2} \pi_{1}}{\partial a^{2}}=\frac{2401(1-\delta)}{93312(1+\delta)^{2}}>0$ ) is not in the area of positive prices.

${ }^{25}$ Indeed $\frac{1}{2}-\frac{\sqrt{1-\delta^{2}}}{(1-\delta) 2 \sqrt{5}}<\frac{1}{3+\delta}$ for all $\delta \in(-1,1)$.

${ }^{26}$ When $1-a^{*}-b^{*} \geq 0$ this is done by choosing larger values of $a$, i.e. by moving to the right of Firm 2 ; when $1-a^{*}-b^{*} \leq 0$ this is done by selecting lower values of $a$, i.e. by moving to the left of Firm 2 .
} 


\section{REFERENCES}

AlCACER, J., AND W. CHUNG (2007): "Location strategies and knowledge spillovers," Management science, 53(5), 760-776.

Anderson, S. P., AND D. J. Neven (1991): “Cournot competition yields spatial agglomeration,” International Economic Review, pp. 793-808.

Bowley, A. L. (1924): Mathematical groundwork of economics. California Press, Oxford.

BRANDER, J. A., AND B. J. SPENCER (2015): "Endogenous horizontal product differentiation under bertrand and cournot competition: Revisiting the bertrand paradox," Discussion paper, National Bureau of Economic Research.

Colombo, L., AND H. DAWID (2014): "Strategic location choice under dynamic oligopolistic competition and spillovers," Journal of Economic Dynamics and Control, 48, 288-307.

D’ Aspremont, C., J. J. Gabszewicz, AND J.-F. Thisse (1979): “On Hotelling's" Stability in competition"," Econometrica: Journal of the Econometric Society, pp. 1145-1150.

DE FrajA, G., AND G. NORMAN (1993): "Product differentiation, pricing policy and equilibrium,”Journal of Regional Science, 33(3), 343-363.

De Palma, A., V. Ginsburgh, Y. Y. Papageorgiou, And J.-F. Thisse (1985): "The principle of minimum differentiation holds under sufficient heterogeneity," Econometrica: Journal of the Econometric Society, pp. 767-781.

De Palma, A., M. Labbé, J.-F. Thisse, And G. Norman (1986): “On the existence of price equilibria under mill and uniform delivered price policies," Spatial pricing and differentiated markets, pp. 30-42.

DiXIT, A. (1979): "A model of duopoly suggesting a theory of entry barriers," The Bell Journal of Economics, pp. 20-32.

ECONOMIDES, N., ET AL. (1986): "Minimal and maximal product differentiation in Hotellings duopoly," Economics Letters, 21(1), 67-71.

Gracia, A., J. BARREIRO-Hurlé, AND B. L. GALÁN (2014): "Are local and organic claims complements or substitutes? A consumer preferences study for eggs," Journal of Agricultural Economics, 65(1), 49-67.

GUPTA, B., D. PAL, AND J. SARKAR (1997): "Spatial Cournot competition and agglomeration in a model of location choice," Regional Science and Urban Economics, 27(3), 261-282.

Hamilton, J. H., J.-F. Thisse, A. Weskamp, et Al. (1987): Spatial discrimination: Bertrand vs. Cournot in a model of location choice. INSEAD.

Hotelling, H. (1990): "Stability in competition," in The Collected Economics Articles of Harold Hotelling, pp. 50-63. Springer.

IRMEN, A., J.-F. ThISSE, ET AL. (1998): "Competition in multi-characteristics spaces: Hotelling was almost right," Journal of economic theory, 78(1), 76-102.

JOHnson, J. P., AND D. P. MYATT (2006): "On the simple economics of advertising, marketing, and product design," American Economic Review, 96(3), 756-784.

LANCASTER, K. (1971): Consumer demand: A new approach. Columbia University Press.

LANCASTER, K. J. (1966): “A new approach to consumer theory,” Journal of political economy, 74(2), $132-157$.

LANCASTER Kelvin, J. (1975): “Socially optimal product différenciation,” American Economic Review, $65,567-585$.

Meas, T., W. Hu, M. T. Batte, T. A. Woods, And S. ERnst (2014): "Substitutes or complements? Consumer preference for local and organic food attributes," American Journal of Agricultural Economics, 97(4), 1044-1071.

Neven, D., AND J.-F. Thisse (1989): “Choix des produits: concurrence en qualité et en variété,” Annales d'Économie et de Statistique, pp. 85-112.

PAL, D. (1998): "Does Cournot competition yield spatial agglomeration?,” Economics Letters, 60(1), 4953.

PEITZ, M. (1997): "Models à la Lancaster and à la Hotelling: when they are the same," Economics Letters, $54(2), 147-154$

SINGH, N., AND X. Vives (1984): "Price and quantity competition in a differentiated duopoly," The RAND Journal of Economics, pp. 546-554.

Tremblay, C. H., AND V. J. TRemblay (2011): “The Cournot-Bertrand model and the degree of product differentiation," Economics Letters, 111(3), 233-235. 